\title{
Special Issue Featuring Papers from the International Thermal Spray Conference (ITSC) 2021
}

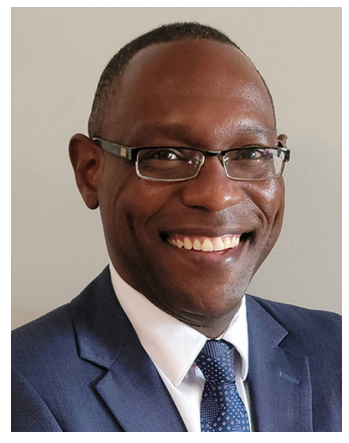

André McDonald

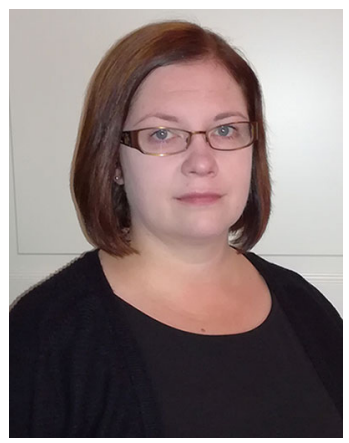

Heli Koivuluoto

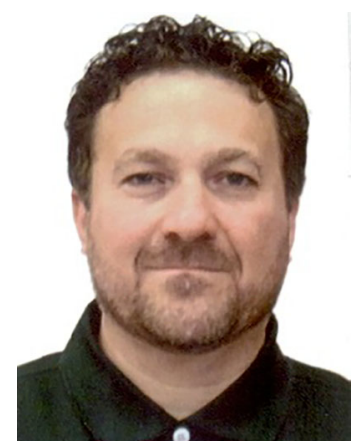

Fardad Azarmi

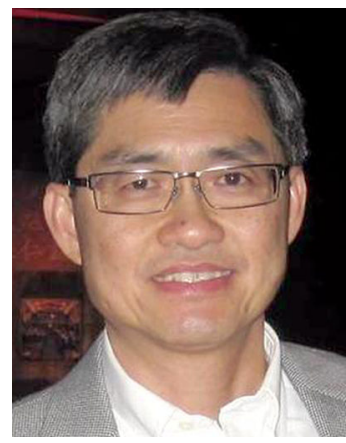

Yuk-Chiu Lau

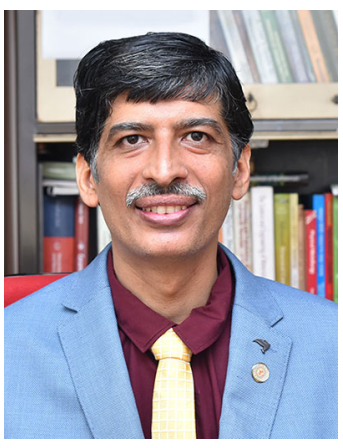

Kantesh Balani

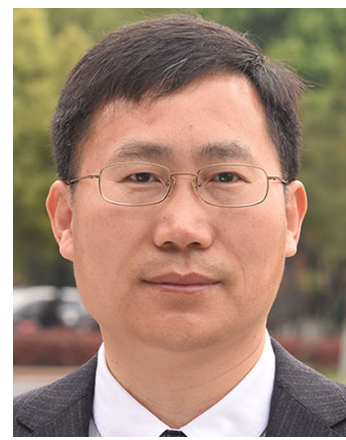

Hua Li

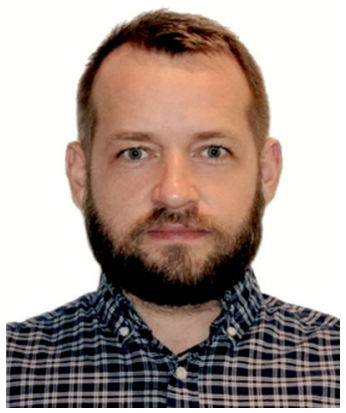

Jan Cizek

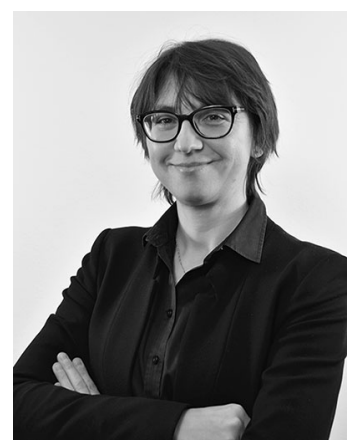

Filofteia-Laura Toma
The COVID-19 pandemic and government restrictions continued to have marked impact on the delivery of the flagship International Thermal Spray Conference. As a result, the International Thermal Spray Conference 2021 (ITSC 2021) was delivered via a virtual platform. Conference manuscripts were submitted, and this special issue 
of the Journal of Thermal Spray Technology (JTST) presents a selection of those manuscripts expanded into fulllength, peer-reviewed articles. The conference was held May 24-28, 2021, and was organized by the ASM International Thermal Spray Society (TSS).

Similar to previous years when the conference was held in person, it drew participation from international academic, government, and industry stakeholders to exhibitions, industry forum, and technical presentations on thermal spraying, coatings, and novel next-generation hybridization of thermal spraying with other surface fabrication processes. The topics covered included subjects on fundamental and applied aspects of the science and engineering of thermal spray processing, coatings, modelling and simulation, diagnostics, equipment and supplies, and new markets for thermal-sprayed coatings and equipment. Next-generation topics, such as additive manufacturing, life-cycle analysis for environmental impact, and new coating and materials development, were also explored. The development of advanced coatings by way of thermal spray processes such as suspension plasma spraying, highvelocity-oxy-fuel spraying, high velocity air-fuel spraying, and cold spraying were featured prominently. The results of novel industrial applications were also a key feature of the conference. The selected articles in this special issue highlight cutting-edge research and knowledge mobilization in antiviral response of coatings, cold-spray metallization of polymers, in-flight thermal-sprayed particle diagnostics, numerical simulation of coating stress and damage under loading, additive manufacturing using cold spraying, and development of new high-entropy alloy coating materials.

The submitted articles and the delivered presentations at the ITSC showcased the innovative and applied nature of the research activities that are current in the field of thermal spraying. This special issue of the JTST presents an extended version of the articles, selected from the 63 invited submissions of ITSC 2021 Proceedings, and which provide a detailed and substantive treatment of the research results that is typical of high-quality peer-reviewed journal manuscripts. This volume will be a valuable source of information and guidance for scientists, engineers, and students to reference as they work to develop advanced thermal-sprayed coatings and novel deposition processes to address current and future industry needs.
We sincerely thank the reviewers for the time that they took to conduct thorough and high-quality reviews. We also express our gratitude to the authors for their impactful contributions and thoughtful responses to the comments of the reviewers.

\section{Lead Editor}

\section{André McDonald}

University of Alberta

Edmonton, AB, Canada

Guest Editors

Fardad Azarmi

North Dakota State University

Fargo, ND, USA

Kantesh Balani

Indian Institute of Technology Kanpur

Kanpur, India

\section{Jan Cizek}

Institute of Plasma Physics

The Czech Academy of Sciences

Prague, Czech Republic

\section{Heli Koivuluoto}

Tampere University of Technology

Tampere, Finland

\section{Yuk-Chiu Lau}

GE Power and Water (retired)

Schenectady, NY, USA

\section{Hua Li}

Ningbo Institute of Materials Technology and Engineering (CAS)

Ningbo, Zhejiang, China

\section{Filofteia-Laura Toma}

Fraunhofer Institute-IWS

Dresden, Germany 